\title{
The Darboux system: Finite-rank constraints and Darboux transformations
}

\author{
Franciso Guil and Manue Man as $^{\mathrm{a})}$ \\ Departamento de Fica Térica, Universidad Complutense, E28040-Madrid, Spain
}

(Received 3 January 1997; accepted for publication 7 May 1997)

The exponential solutions of the Darboux equations for conjugate nets is considered. It is shown that rank-one constraints over the right derivatives of invertible operators on an arbitrary linear space give solutions of the Darboux system, which can be understood as a vectorial Darboux transformation of the exponential background. The method is extended further to obtain vectorial Darboux transformations of the Darboux system. (C) 1997 American Institute of Physics.

[S0022-2488(97)00210-7]

\section{INTRODUCTION}

This paper is devoted to the analysis of the Darboux equations for conjugate nets and its relation with certain finite-rank constraints on elements in the group $\operatorname{GL}(V)$. The exponential solutions of these equations are deformed, through the mentioned connection, to new solutions. As a byproduct we obtain vectorial Darboux transformations for the Darboux equations for conjugate nets.

In the context of differential geometry the Darboux equations describe conjugates nets in an $N$-dimensional surface as is explained in Ref. 1. This fact explains its relevance in this discipline and its appearance in classical texts on the subject; see, for example, Refs. 2 and 3. In integrable system theory a matrix version of it was proposed and solved by Zakharov and Manakov in Ref. 4. The Darboux equations for conjugate nets appear also as the first flow in the $N$-component Kadomtsev-Petviasvili hierarchy. The Darboux equations are associated with the $N$-wave interaction (see Ref. 5) and, in particular, for triply orthogonal systems, to the equations describing the resonant interaction of three waves ${ }^{6-10}$ which has been widely applied in the context of fluid dynamics, nonlinear optical phenomena, ${ }^{11}$ and plasma physics ${ }^{12}$ (see Refs. 13 and 14).

Given $N(N-1)$ functions $\left\{p_{i j}\right\}_{i, j=1, \ldots, N}$ depending on $N$ curvilinear coordinates $x_{1}, \ldots, x_{N}$, the Darboux system is

$$
\partial_{k} p_{i j}+p_{i k} p_{k j}=0, \text { for distinct } i, j, k=1, \ldots, N \text {, }
$$

where $\partial_{i}:=\partial / \partial x_{i}$. These equations are the compatibility conditions for the linear system

$$
\partial_{j} F_{i}+p_{i j} F_{j}=0, \text { for } i \neq j,
$$

or for the dual linear system for $\widetilde{F}_{i}, i=1, \ldots, N$,

$$
\partial_{j} \widetilde{F}_{i}+p_{j i} \widetilde{F}_{j}=0, \text { for } i \neq j
$$

Equations (1) have two obvious symmetries. First, we consider changes in the amplitude. The action in the moduli space is

$$
p_{i j}\left(x_{1}, \ldots, x_{N}\right) \mapsto \exp \left(a_{i}\left(x_{i}\right)-a_{j}\left(x_{j}\right)\right) p_{i j}\left(x_{1}, \ldots, x_{N}\right)
$$

${ }^{a)}$ Electronic mail: manuel@dromos.fis.ucm.es 
for arbitrary functions $a_{i}\left(x_{i}\right), i=1,2, \ldots, N$. Another symmetry is a scaling transformation defined by any set of nonzero numbers $\left\{s_{i}\right\}_{i=1,2, \ldots N}$ as

$$
p_{i j}\left(x_{1}, \ldots, x_{N}\right) \mapsto s_{i} s_{j} p_{i j}\left(x_{1} / s_{1}^{2}, \ldots, x_{N} / s_{N}^{2}\right)
$$

that provides an action on the solution space.

In this paper a special role will be played by the exponential solutions of the Darboux equations (1),

$$
p_{i j}^{(0)}\left(x_{1}, \ldots, x_{N}\right)=\lambda_{i j} \exp \left(-\sum_{k \neq i} x_{k} \mu_{k i}+\sum_{k \neq j} x_{k} \mu_{k j}\right),
$$

where $\lambda_{i j}$ and $\mu_{i j}$ are subject to

$$
\lambda_{i j}\left(\mu_{k i}-\mu_{k j}\right)=\lambda_{i k} \lambda_{k j}
$$

for $i, j, k=1, \ldots, N$ and different.

Observe that there is a compatibility condition over the amplitudes $\lambda_{i j}$, namely

$$
\lambda_{i j} \lambda_{j k} \lambda_{k i}+\lambda_{i k} \lambda_{k j} \lambda_{j i}=0
$$

for all $i, j, k$ different, and if the $\lambda$ 's do not vanish this equation itself gives the possible $\lambda$ 's that when plugged into Eq. (7) give the differences $\mu_{k i}-\mu_{k j}$. That only the differences are fixed is a consequence of the symmetry of the Darboux system defined in (4). Indeed, given an exponential solution with parameters $\left\{\lambda_{i j}, \mu_{i j}\right\}$, then the set $\left\{\lambda_{i j}, \mu_{i j}+a_{i}\right\}$ defines another possible exponential solution [here we have taken the functions $\left.a_{i}\left(x_{i}\right)=a_{i} x_{i}\right]$. Observe also that Eqs. (7) are invariant under the substitution $\left\{\lambda_{i j}, \mu_{i j}\right\} \mapsto\left\{s_{i} s_{j} \lambda_{i j}, s_{i}^{2} \mu_{i j}\right\}$, a consequence of the symmetry transformation (5).

The motivation of this paper comes from our previous work. ${ }^{15-18}$ The main idea in it is to consider rank-one constraints on the right derivatives of certain invertible operators. This was done in Ref. 15 for the Kadomtsev-Petviashvili equation and extended to the Davey-Stewartson equations in Refs. 16 and 18. In Ref. 17 we studied a deformation of the dromion solution of DSI arising naturally from our method. In connection with this we mention Refs. 19-22, where vectorial Darboux transformations for the Davey-Stewartson, nonlinear Schrödinger, multidimensional quadrilateral lattices, and Manin-Radul super Korteweg-de Vries equation were given, respectively; in Ref. 23 the Wronskian scheme, appearing as a byproduct of our work on DaveyStewartson II, was used to give a detailed analysis of interesting novel solutions of this equation.

Section II is devoted to the study of these rank-one constraints, which in this case are connected with the exponential solutions of (1). Next, in Sec. III we show that the solutions obtained in Sec. II generalize to a deformation of the exponential solutions. This motivates a further extension in Sec. IV where a vectorial Darboux transformation for the Darboux system is given. For any solution we consider vector solutions of the associated Lax pairs in terms of which we construct Grammian-type determinants that allow us to give large families of new solutions.

\section{RANK-ONE CONSTRAINTS AND THE DARBOUX SYSTEM}

In this section we shall show how invertible operators can give solutions to the Darboux system given by Eqs. (1). Consider a function $\psi\left(x_{1}, \ldots, x_{N}\right)$ of the $N$ variables $\left\{x_{1}, \ldots, x_{N}\right\}$ taking values on $\operatorname{GL}(V)$, the set of invertible operators on some complex linear space $V$. On this function we impose some differential constraints, namely its right derivatives are of the following form:

$$
\partial_{i} \psi \cdot \psi^{-1}=A_{i}+e_{i} \otimes \alpha_{i}, \quad i=1, \ldots, N,
$$


where $A_{i}$ are constant operators on $V, e_{1}, \ldots, e_{N}$ are $N$ independent constant vectors on $V$, and $\alpha_{i}\left(x_{1}, \ldots, x_{N}\right)$ takes values on the set of linear functionals over $V$, the dual space of covectors, $V^{*}$. Now, we must take care of the compatibility conditions for Eqs. (9). In order to have a set of closed conditions we require the following.

(i) The operators $A_{i}$ must commute among them.

(ii) The image of the operator $A_{i}$ when acting on the vector $e_{j}, i \neq j$, must be expanded by $e_{i}$ and $e_{j}$ :

$$
A_{i} e_{j}=\lambda_{i j} e_{i}+\mu_{i j} e_{j}, \quad i \neq j
$$

The coefficients $\lambda_{i j}$ and $\mu_{i j}$ are not completely free, indeed there is a further compatibility condition: $\left[A_{i}, A_{j}\right] e_{k}=0$ for $i, j, k$ different. This condition is just Eq. (7). Concrete realizations of such operators are easily constructed in any space $V$ although we do not need the explicit form of them. The compatibility conditions arising from the rank-one constraints for the right derivatives of $\psi$ are

$$
\left(\partial_{j}-\mu_{j i}\right) \alpha_{i}+\pi_{i j} \alpha_{j}+\alpha_{i} A_{j}=0
$$

where

$$
\pi_{i j}:=\lambda_{i j}+\left\langle\alpha_{i}, e_{j}\right\rangle
$$

The contraction of Eq. (10) with the vector $e_{k}, k \neq i, j$, gives

$$
\left(\partial_{j}+\mu_{j k}-\mu_{j i}\right) \pi_{i k}+\pi_{i j} \pi_{j k}=0,
$$

and these equations can be simplified by defining

$$
p_{i j}:=\exp \left(-\sum_{k \neq i} x_{k} \mu_{k i}+\sum_{k \neq j} x_{k} \mu_{k j}\right) \pi_{i j} \text {, }
$$

to obtain Eqs. (1) for the functions $p_{i j}$.

Therefore, we have shown how rank-one constraints over the right derivatives of an invertible operator give rise to solutions of the Darboux system which in turn implies that solving our constrained system allows us to find solutions of the Darboux.

To construct suitable operators $\psi$ we introduce the following linear functionals on $V$ :

$$
\beta_{i}:=\exp \left(-\sum_{k \neq i} x_{k} \mu_{k i}\right) \alpha_{i} \psi,
$$

so that $\left(\partial_{i}-A_{i}\right) \psi=\exp \left(\sum_{k \neq i} x_{k} \mu_{k i}\right) e_{i} \otimes \beta_{i}$ and the compatibility conditions $\left[\partial_{i}-A_{i}, \partial_{j}-A_{j}\right] \psi=0$ read

$$
\partial_{j} \beta_{i}+p_{i j}^{(0)} \beta_{j}=0, \quad i \neq j
$$

with $p_{i j}^{(0)}$ as given in Eq. (6).

We also introduce $\psi_{0}:=\exp \left(\sum_{i} x_{i} A_{i}\right), \varphi:=\psi_{0}^{-1} \cdot \psi$, and

$$
b_{i}:=\exp \left(\sum_{k \neq i} x_{k} \mu_{k i}\right) \psi_{0}^{-1} e_{i} .
$$

Then, the rank-one conditions (9) on the right derivatives of $\psi$ determine that 


$$
\partial_{i} \varphi=b_{i} \otimes \beta_{i}
$$

Conversely, given operators $A_{i}$ as prescribed before and the related objects $\psi_{0}$ and $b_{i}$, as well as solutions $\beta_{i}$ to Eq. (11), we can integrate Eq. (12) and then obtain $\psi=\psi_{0} \cdot \varphi$ as required.

Summarizing, we can construct solutions of the Darboux system (1) as follows:

Theorem 1: Given $N$ commuting operators $A_{1}, \ldots, A_{N}$ on a complex linear space $V, N$ independent linear vectors $e_{1}, \ldots, e_{N}$, such that

$$
A_{i} e_{j}=\lambda_{i j} e_{i}+\mu_{i j} e_{j}, \quad i \neq j
$$

for $i \neq j$, where $\lambda_{i j}$ and $\mu_{i j}$ satisfy

$$
\left(\mu_{k i}-\mu_{k j}\right) \lambda_{i j}=\lambda_{i k} \lambda_{k j},
$$

with $i, j, k=1, \ldots, N$ different, we define the $N$ vector functions

$$
b_{i}=\exp \left(\sum_{k \neq i} x_{k} \mu_{k i}\right) \psi_{0}^{-1} e_{i}, \quad i=1, \ldots, N,
$$

where $\psi_{0}=\exp \left(\sum_{i} x_{i} A_{i}\right)$ and the linear covector functions $\beta_{i}, i=1, \ldots, N$, subject to

$$
\partial_{j} \beta_{i}+p_{i j}^{(0)} \beta_{j}=0, \quad i \neq j
$$

with $p_{i j}^{(0)}=\lambda_{i j} \exp \left(-\sum_{k \neq i} x_{k} \mu_{k i}+\sum_{k \neq j} x_{k} \mu_{k j}\right)$. If we define an invertible operator $\varphi$ by the compatible equations

$$
\partial_{i} \varphi=b_{i} \otimes \beta_{i}
$$

then the functions

$$
p_{i j}:=p_{i j}^{(0)}+\left\langle\beta_{i}, \varphi^{-1} b_{j}\right\rangle
$$

solve the Darboux system

$$
\partial_{j} p_{i k}+p_{i j} p_{j k}=0
$$

Notice the different role played by the $b$ 's and the $\beta$ 's. The $\beta_{i}$ are simply solutions of Eq. (11) while the definition of the $b_{i}$ is given in terms of the $A_{i}$ and the vectors $e_{i}$. Nevertheless, both need the coefficients $\{\lambda, \mu\}$ defined by Eqs. (7). However, one can show that in fact the $b$ 's do satisfy analogous equations to those defining the $\beta$ 's, namely

$$
\partial_{j} b_{i}+p_{j i}^{(0)} b_{j}=0
$$

which can be considered adjoint to (11).

We can also seek wave functions solving the Lax pair or its dual for the solutions given in the previous theorem:

Proposition 1: The functions

$$
\begin{aligned}
& F_{i}=\beta_{i} \varphi^{-1}, \\
& \widetilde{F}_{i}=\varphi^{-1} b_{i},
\end{aligned}
$$

satisfy Eqs. (2) and (3), respectively, where the expression for $p_{i j}$ is given in (13).

The proof is just a simple check. First take the derivative with respect to $x_{j}$ to $F_{i}$ 


$$
\partial_{j} F_{i}=\left(\partial_{j} \beta_{i}\right) \varphi^{-1}-F_{i}\left(\partial_{j} \varphi \cdot \varphi^{-1}\right),
$$

then use Eqs. (11) and (12) to evaluate the derivatives of $\beta_{i}$ and $\varphi$ and to obtain Eq. (2) with $p_{i j}$ defined in (13). For $\widetilde{F}_{i}$ we proceed in an analogous manner:

$$
\partial_{j} \widetilde{F}_{i}=-\left(\varphi^{-1} \cdot \partial_{j} \varphi\right) \widetilde{F}_{i}+\varphi^{-1} \partial_{j} b_{i},
$$

but now we need Eqs. (14) and (12).

\section{DEFORMATIONS OF THE EXPONENTIAL SOLUTIONS FOR THE DARBOUX SYSTEM}

Equations (14) do not characterize the $b$ 's, but one can easily show that in order to construct solutions of the Darboux system we only need solutions of the linear Eqs. (14) and (11). Suppose that we have $b_{i}, i=1, \ldots, N$, vector functions satisfying Eqs. (14); $\beta_{i}, i=1, \ldots, N$, linear functionals that are solutions of Eqs. (11); $\varphi$ a solution of (12); and define $p_{i j}$ as in (13). Then, we can evaluate the derivative of $p_{i j}$ with respect to $x_{k}$ to obtain

$$
\begin{aligned}
\partial_{k} p_{i j}= & \left(-\mu_{k i}+\mu_{k j}\right) \lambda_{i j} \exp \left(-\sum_{k \neq i} x_{k} \mu_{k i}+\sum_{k \neq j} x_{k} \mu_{k j}\right) \\
& +\left\langle\partial_{k} \beta_{i}, \varphi^{-1} b_{j}\right\rangle-\left\langle\beta_{i}, \varphi^{-1}\left(\partial_{k} \varphi\right) \varphi^{-1} b_{j}\right\rangle+\left\langle\beta_{i}, \varphi^{-1} \partial_{k} b_{j}\right\rangle,
\end{aligned}
$$

and, using Eqs. (7), (11), (14), and (12), we find out

$$
\partial_{k} p_{i j}=-p_{i k}^{(0)} p_{k j}^{(0)}-p_{i k}^{(0)}\left\langle\beta_{k}, \varphi^{-1} b_{j}\right\rangle-p_{k j}^{(0)}\left\langle\beta_{i}, \varphi^{-1} b_{k}\right\rangle-\left\langle\beta_{i}, \varphi^{-1} b_{k}\right\rangle\left\langle\beta_{k}, \varphi^{-1} b_{j}\right\rangle=-p_{i k} p_{k j},
$$

as desired.

Moreover, we can construct wave functions and its adjoints as before.

Summarizing,

Theorem 2: (i) Given $\left\{\lambda_{i j}, \mu_{i j}\right\}_{i, j=1}{ }_{i \neq j}, \ldots,{ }_{N} \subset \mathrm{C}$ subject to

$$
\left(\mu_{k i}-\mu_{k j}\right) \lambda_{i j}=\lambda_{i k} \lambda_{k j}, \quad i, j, k=1, \ldots, N \text { and distinct },
$$

the exponential solutions of the Darboux system (1) are

$$
p_{i j}^{(0)}=\lambda_{i j} \exp \left(-\sum_{k \neq i} x_{k} \mu_{k i}+\sum_{k \neq j} x_{k} \mu_{k j}\right) .
$$

(ii) Deformations of the exponential solutions are constructed as follows: Take vector functions $b_{i}\left(x_{1}, \ldots, x_{N}\right) \in V, i=1, \ldots, N$, where $V$ is a complex linear space, solutions of

$$
\partial_{j} b_{i}+p_{j i}^{(0)} b_{j}=0,
$$

define covectors $\beta_{i}\left(x_{1}, \ldots, x_{N}\right) \in V^{*}, i=1, \ldots, N$, subject to

$$
\partial_{j} \beta_{i}+p_{i j}^{(0)} \beta_{j}=0, \quad i \neq j,
$$

and integrate the compatible equations

$$
\partial_{i} \varphi=b_{i} \otimes \beta_{i} .
$$

Then the set of functions 


$$
p_{i j}:=p_{i j}^{(0)}+\left\langle\beta_{i}, \varphi^{-1} b_{j}\right\rangle
$$

solves

$$
\partial_{j} p_{i k}+p_{i j} p_{j k}=0 .
$$

The functions $F_{i}=\beta_{i} \varphi^{-1}$, which are $V^{*}$-valued, and $\widetilde{F}_{i}=\varphi^{-1} b_{i}$, which are $V$-valued functions, satisfy the linear equations

$$
\begin{aligned}
& \partial_{j} F_{i}+p_{i j} F_{j}=0, \\
& \partial_{j} \widetilde{F}_{i}+p_{j i} \widetilde{F}_{j}=0,
\end{aligned}
$$

the Lax pairs (2) and (3), respectively.

The solution shown in part (ii) is a deformation of the exponential solutions $p_{i j}^{(0)}$ of (i) because they are a particular case of $p_{i j}$ when $b_{i}=0$ and $\beta_{i}=0, i=1, \ldots, N$. Thus, these exponential solutions can be considered as the starting solutions we dress in terms of which we obtain the families of solutions described above, and hence as our vacuum solutions.

Observe that in the previous section we found explicit solutions, the $b$ 's for the associated linear system. The $\beta$ 's can be taken in a similar way. This is quite convenient if one wants to compute the deformation of the exponential background. The solutions to the linear system are already provided, which is the main drawback of Darboux transformations to solve the associated linear system for nonzero backgrounds.

\section{VECTORIAL DARBOUX TRANSFORMATIONS FOR THE DARBOUX SYSTEM}

Darboux transformations were first considered by Moutard and Darboux. ${ }^{24,25}$ Here we extend the results of Darboux to a vectorial Darboux transformation of the Darboux system.

For a given solution $p_{i j}$ of Eqs. (1) and a complex linear space $V$ we consider solutions $b_{i}\left(x_{1}, \ldots, x_{N}\right) \in V, i=1, \ldots, N$, and $\beta_{i}\left(x_{1}, \ldots, x_{N}\right) \in V^{*}, i=1, \ldots, N$, of

$$
\begin{aligned}
& \partial_{j} b_{i}+p_{j i} b_{j}=0, \\
& \partial_{j} \beta_{i}+p_{i j} \beta_{j}=0 .
\end{aligned}
$$

By virtue of the previous linear systems the following equation holds:

$$
\partial_{j}\left(b_{i} \otimes \beta_{i}\right)=\partial_{i}\left(b_{j} \otimes \beta_{j}\right) .
$$

This implies the existence of a local potential, say $\varphi$, such that

$$
\partial_{j} \varphi=b_{i} \otimes \beta_{i} .
$$

As the operator $\varphi$ is defined up to a constant, we suppose that it can be chosen to be invertible, $\varphi\left(x_{1}, \ldots, x_{N}\right) \in \operatorname{GL}(V)$. With this operator we construct new functions $\hat{b}_{i}$ and $\hat{\beta}_{i}$ as follows:

$$
\hat{b}_{i}:=\varphi^{-1} b_{i} \quad \text { and } \hat{\beta}_{i}:=\beta_{i} \varphi^{-1}, \quad i=1, \ldots, N .
$$

If we define now

$$
\hat{p}_{i j}:=p_{i j}+\left\langle\beta_{i}, \hat{b}_{j}\right\rangle=p_{i j}+\left\langle\hat{\beta}_{i}, b_{j}\right\rangle=p_{i j}+\left\langle\beta_{i}, \varphi^{-1} b_{j}\right\rangle=p_{i j}+\left\langle\hat{\beta}_{i}, \varphi \hat{b}_{j}\right\rangle,
$$

we immediately see that 


$$
\begin{aligned}
\partial_{j} \hat{b}_{i}+\hat{p}_{j i} \hat{b}_{j}=0, \\
\partial_{j} \hat{\beta}_{i}+\hat{p}_{i j} \hat{\beta}_{j}=0
\end{aligned}
$$

so that $\hat{p}_{i j}$ is a solution again

Theorem 3: Let $p_{i j}$ be a solution of Eqs. (1) and define $b_{i}$ and $\beta_{i}$ as solutions of the linear systems

$$
\begin{aligned}
\partial_{j} b_{i}+p_{j i} b_{j} & =0, \\
\partial_{j} \beta_{i}+p_{i j} \beta_{j} & =0,
\end{aligned}
$$

with $b_{i}, i=1, \ldots, N$, taking values in some complex linear space, and $\beta_{i}, i=1, \ldots, N$, in its dual. If $\varphi$ is an invertible solution of the compatible equations

$$
\partial_{i} \varphi=b_{i} \otimes \beta_{i},
$$

then

$$
\hat{p}_{i j}=p_{i j}+\left\langle\beta_{i}, \varphi^{-1} b_{j}\right\rangle
$$

is another solution of Eqs. (1).

Proof: The result follows from the considerations previous to the theorem. Nevertheless, a direct check is easy:

$$
\begin{aligned}
\partial_{k} \hat{p}_{i j} & =\partial_{k} p_{i j}+\left\langle\partial_{k} \beta_{i}, \varphi^{-1} b_{j}\right\rangle-\left\langle\beta_{i}, \varphi^{-1}\left(\partial_{k} \varphi\right) \varphi^{-1} b_{j}\right\rangle+\left\langle\beta_{i}, \varphi^{-1} \partial_{k} b_{j}\right\rangle \\
& =-p_{i k} p_{k j}-p_{i k}\left(\hat{p}_{k j}-p_{k j}\right)-\left(\hat{p}_{i k}-p_{i k}\right)\left(\hat{p}_{k j}-p_{k j}\right)-p_{k j}\left(\hat{p}_{i k}-p_{i k}\right) \\
& =-\hat{p}_{i k} \hat{p}_{k j} .
\end{aligned}
$$

This theorem allows us to deform a given solution by solving the associated linear problem. We see that the solutions are expressed in terms of Grammian determinants of the $b$ 's and $\beta$ 's. The function $\varphi$ can be expressed as

$$
\varphi\left(x_{1}, \ldots, x_{N}\right)=C+\int_{\gamma}\left(\sum_{i=1, \ldots, N} \partial x_{i} b_{i} \otimes \beta_{i}\right),
$$

where $C$ is any constant operator in $V$ and $\gamma$ is an adequate path in $\mathrm{C}^{N}$ with end point $x_{1}, \ldots, x_{N}$, such that $\varphi$ has a nonvanishing determinant and $\tau=\operatorname{det} \varphi$ is the principal tau function. If we define the operators $\varphi_{i j}:=\varphi+b_{j} \otimes\left(\beta_{i}-\delta_{i} \varphi\right)$, with $\delta_{i}\left(x_{1}, \ldots, x_{N}\right) \in V^{*}$ such that $\left\langle\delta_{i}, b_{j}\right\rangle=\delta_{i j}$, and denote their determinants by $\tau_{i j}=\operatorname{det} \varphi_{i j}$, the associated tau functions, we arrive at the expressions $p_{i j}=\tau_{i j} / \tau$.

Let us mention that this vectorial Darboux transformation can be obtained through a dim $V$-step interation of a corresponding binary Darboux transformation, this last one being a composition of two standard Darboux transformations. For the three-wave resonant interaction a similar result can be found in Ref. 26 under the name of Kaup system.

${ }^{1}$ G. Darboux, Leçons sur la Theórie Générale des Surfaces IV, Liv. VIII (Gauthier-Villars, Paris, 1896), Chap. XII.

${ }^{2}$ L. P. Eisenhart, A Treatise on the Differential Geometry of Curves and Surfaces (Ginn, Boston, 1909); An Introduction to Differential Geometry with use of Tensor Analysis (Princeton U. P., Princeton, NJ, 1940).

${ }^{3}$ A. R. Forsyth, Lectures on the Differential Geometry of Curves and Surfaces (Cambridge U.P., Cambridge, 1912).

${ }^{4}$ V. E. Zakharov and S. E. Manakov, Funct. Anal. Appl. 19, 89 (1985). 
${ }^{5}$ M. J. Ablowitz and P. A. Clarkson, Solitons, Nonlinear Evolution Equations and Inverse Scattering, London Math. Soc. Lec. Not. Ser. 149 (Cambridge U.P., Cambridge, 1989).

${ }^{6}$ V. E. Zakharov, Sov. Phys. Dokl. 21, 322 (1976).

${ }^{7}$ V. E. Zakharov and A. B. Shabat, Funct. Anal. Appl. 8, 226 (1974).

${ }^{8}$ H. Cornille, J. Math. Phys. 20, 1653 (1979).

${ }^{9}$ M. J. Ablowitz and R. Haberman, Phys. Rev. Lett. 35, 1185 (1975).

${ }^{10}$ D. J. Kaup, Physica D 1, 45 (1981); 3, 374 (1981); J. Math. Phys. 22, 1176 (1981).

${ }^{11}$ J. A. Armstrong, N. Bloembergen, J. Ducuing, and P. S. Pershan, Phys. Rev. 127, 1918 (1962).

${ }^{12}$ B. Coppi, M. N. Rosenbluth, and R. N. Sudan, Ann. Phys. 55, 207 (1969).

${ }^{13}$ V. E. Zakharov, S. L. Musher, and A. M. Rubenckik, Phys. Rep. 129, 285 (1986).

${ }^{14}$ D. J. Kaup, A. Reiman, and A. Bers, Rev. Mod. Phys. 51, 275 (1979).

${ }^{15}$ F. Guil, M. Mañas, and G. Álvarez, Phys. Lett. A 190, 49 (1994).

${ }^{16}$ F. Guil and M. Mañas, J. Phys. A 28, 1713 (1995).

${ }^{17}$ F. Guil and M. Mañas, Phys. Lett. A 209, 29 (1995).

${ }^{18}$ F. Guil and M. Mañas, J. Phys. A 29, 641 (1996).

${ }^{19}$ F. Guil and M. Mañas, Phys. Lett. A 217, 1 (1996).

${ }^{20}$ M. Mañas, J. Phys. A 29, 7721 (1996).

${ }^{21}$ M. Mañas, A. Doliwa, and P. M. Santini, Phys. Lett. A 233, 365 (1997).

${ }^{22}$ Q. P. Liu and M. Mañas, Phys. Lett. B 394, 337 (1997).

${ }^{23}$ M. Mañas and P. M. Santini, Phys. Lett. A 227, 325 (1997).

${ }^{24}$ Th. F. Moutard, C. R. Acad. Sci. Paris 80, 729 (1875); J. l'Ecole Polytech. 45, 1 (1878).

${ }^{25}$ G. Darboux, C. R. Acad. Sci. Paris 94, 1456 (1882); 94, 120, 158, 1290 (1882).

${ }^{26}$ V. B. Matveev and M. A. Salle, Darboux Transformations and Solitons (Springer, Berlin, 1990). 
Journal of Mathematical Physics is copyrighted by AIP Publishing LLC (AIP). Reuse of AIP content is subject to the terms at: http://scitation.aip.org/termsconditions. For more information, see http://publishing.aip.org/authors/rights-and-permissions. 